\title{
The micromechanics of three-dimensional collagen-I gels
}

\section{Citation}

Stein, Andrew M., David A. Vader, David A. Weitz, and Leonard M. Sander. 2011. "The Micromechanics of Three-Dimensional Collagen-I Gels." Complexity16 (4): 22-28. https:// doi.org/10.1002/cplx.20332.

\section{Permanent link}

http://nrs.harvard.edu/urn-3:HUL.InstRepos:41511298

\section{Terms of Use}

This article was downloaded from Harvard University's DASH repository, and is made available under the terms and conditions applicable to Other Posted Material, as set forth at http:// nrs.harvard.edu/urn-3:HUL.InstRepos:dash.current.terms-of-use\#LAA

\section{Share Your Story}

The Harvard community has made this article openly available.

Please share how this access benefits you. Submit a story.

Accessibility 


\title{
The Micromechanics of Three-Dimensional Collagen-I Gels
}

\author{
ANDREW M. STEIN, ${ }^{1}$ DAVID A. VADER, ${ }^{2}$ DAVID A. WEITZ, ${ }^{2}$ AND LEONARD M. SANDER ${ }^{3}$ \\ ${ }^{1}$ Modeling and Simulation Group, Novartis Institute for BioMedical Research, Cambridge, Massachusetts 02139; \\ ${ }^{2}$ School of Engineering and Applied Sciences, Harvard University, Cambridge, Massachusetts 02138; and \\ ${ }^{3}$ Michigan Center for Theoretical Physics, Department of Physics, University of Michigan, \\ Ann Arbor, Michigan 48109
}

Received 20 December 2009; revised 15 May 2010; accepted 9 June 2010

\begin{abstract}
We study the micromechanics of collagen-I gel with the goal of bridging the gap between theory and experiment in the study of biopolymer networks. Three-dimensional images of fluorescently labeled collagen are obtained by confocal microscopy, and the network geometry is extracted using a 3D network skeletonization algorithm. Each fiber is modeled as an elastic beam that resists stretching and bending, and each crosslink is modeled as torsional spring. The stress-strain curves of networks at three different densities are compared with rheology measurements. The model shows good agreement with experiment, confirming that strain stiffening of collagen can be explained entirely by geometric realignment of the network, as opposed to entropic stiffening of individual fibers. The model also suggests that at small strains, crosslink deformation is the main contributer to network stiffness, whereas at large strains, fiber stretching dominates. As this modeling effort uses networks with realistic geometries, this analysis can ultimately serve as a tool for understanding how the mechanics of fibers and crosslinks at the microscopic level produce the macroscopic properties of the network. ㄷ 2010 Wiley Periodicals, Inc. Complexity 16: 22-28, 2011
\end{abstract}

Key Words: biopolymer; collagen; strain stiffening

\section{INTRODUCTION}

ollagen is the most abundant animal protein [1] and its mechanics have been studied in great detail [2]. It takes on many morphologies, including skin, tendons,

Correspondence to: Andrew M. Stein, Modeling and Simulation Group, Novartis Institute for BioMedical Research, Cambridge, MA 02139 (e-mail:astein@ima.umn.edu) ligaments, individual fibers, and gels. Of particular interest is the mechanics of collagen-I gels, shown in Figure 1(a). These gels provide a relatively simple structure that can be noninvasively observed by confocal microscopy $[3,4]$ and used as a scaffold for growing artificial tissues [5] and as a 3D environment for studying cell motility [6] and tumor invasion [7,8]. A critical first step in understanding these systems is to develop a model for the collagen gel alone. In this article, we give a successful theoretical model of the micromechanics of realistic networks. 


\section{FIGURE 1}

(a)

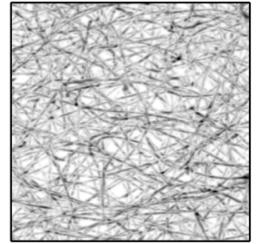

(b)

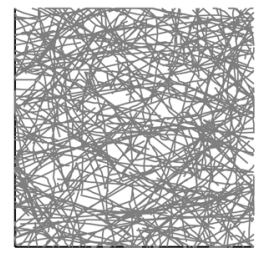

(c)

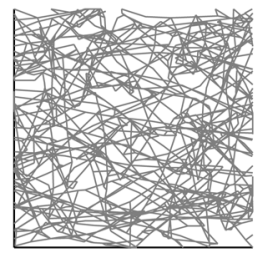

(d)

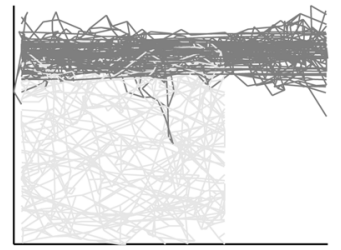

(e)

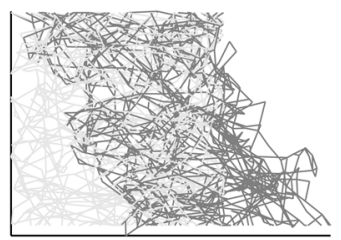

(f)

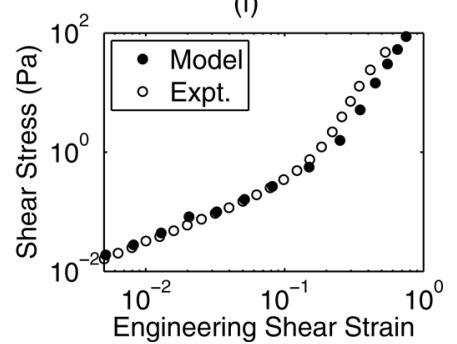

A typical gel $(1.2 \mathrm{mg} / \mathrm{mL}, 25.6 \mu \mathrm{m} \times 25.6 \mu \mathrm{m} \times 25.6 \mu \mathrm{m})$. (a) Maximal intensity projection along the axis that is perpendicular to the focal plane of the microscope. (b) Projection of 3D network extracted by FIRE. (c) Reduced network, where elements that do not contribute to the network stiffness have been removed for improved computational efficiency. (d) Deformation after $50 \%$ tension. (e) Deformation after $50 \%$ shear. (f) Comparison of the stress-strain response between the model and experiment.

Collagen-I gels belong to a class of materials known as biopolymers. Other examples include actin, found in the cytoskeleton, and fibrin, a component of blood clots. Common mechanical features of biopolymers are negative normal stresses under shear [9], significant contraction and alignment as the gel is axially stretched $[10,11]$, and strain stiffening by 2-3 orders of magnitude at large strains, as shown in Figure 1(f). The cause of this strain stiffening is not well understood. Storm et al. [12] attributed strain stiffening in all biopolymer networks exclusively to the pulling out of entropic modes of individual filaments. Their calculation required the assumption that deformations are affine. On the other hand, Heussinger et al. [13, 14] showed how one could deconstruct the network deformation into a set of nonaffine floppy modes. They concluded that accounting for the nonaffinity was necessary in describing the elastic properties of the network. They hypothesize that the nonlinearity comes from a transition from a bending regime to a stretching regime. Onck and coworkers $[15,16]$ have proposed the alternative hypothesis that strain stiffening is due to the rearrangement of the fibers as the network is strained.

Resolving this debate has been difficult as almost all theoretical analysis has been on artificially generated networks in 2D. Whether such artificially generated networks can be used to accurately describe real biopolymer gels has not yet been confirmed. In particular, key results of the above theories rest on assumptions on how the crosslink spacing scales with density. Although a direct relationship has been obtained in two dimensions for random stick networks $[17,18]$, in three dimensions, the scaling relationship will also depend on how the gel polymerizes. Although the nature of collagen polymerization is currently the subject of active research [19], it is still not well understood. Moreover, the few examples of quantitative comparisons to experiment in the literature [12,20,21] are not able to quantitatively fit the full stress-strain response of the gel at varying densities using a single set of parameters. The goal of this research is to develop a model for collagen gel based on three-dimensional images of fluorescently labeled collagen gels at different densities. The images are obtained by confocal microscopy [Figure 1(a)], and the network geometry is extracted using a custom FIbeR Extraction (FIRE) algorithm [Figure 1(b)] [3]. The gel is modeled as a random network of crosslinked fibers, as described below, and the stress-strain response is compared with that measured by an AR-G2 rheometer. Good agreement between model and experiment is obtained by fitting a single parameter, that is, the crosslink stiffness.

\section{EXPERIMENT}

The experiments were defined in more detail previously [3]. Briefly, we labeled bovine collagen type I with the fluorescent molecule TAMRA. Collagen solution at final concentrations of $0.5,1.0$, and $1.5 \mathrm{mg} / \mathrm{mL}$ were prepared, and a Leica SP5 resonant confocal microscope with a 63X 1.2-NA water immersion objective was used to image the collagen samples. Four networks at each density were extracted using the FIRE skeletonization algorithm [3], as shown in Figure 1(b). After applying FIRE, the collagen density was re-estimated in two different ways: by counting the number of bright pixels in the image and by the product of the average fiber crosssectional area $(A=2800 \mathrm{~nm}$ with an assumed radius of $30 \mathrm{~nm}$ $[3,22])$ and total fiber length in the extracted networks. The re-estimated densities of the three networks were $0.5,1.2$, and $1.4 \mathrm{mg} / \mathrm{mL}$. The final step before simulation is to remove dangling portions of the network that do not contribute to its stiffness [Figure 1(c)]. The shear response of the gel was measured using an AR-G2 rheometer with a $4^{\circ}, 40$-mm cone-plate 
geometry with a $109-\mu \mathrm{m}$ gap. Approximately $1.2 \mathrm{~mL}$ of collagen solution was pipetted onto the $37^{\circ} \mathrm{C}$ preheated bottom plate of the rheometer, and the cone was lowered onto the sample. A solvent trap was used to prevent the sample from drying during the measurement.

\section{MODEL}

In the model for the collagen gel, each fiber is treated as an elastic beam that resists stretching and bending and each crosslink is treated as a torsional spring, making it more stiff than a freely rotating pin joint but less stiff than a welded joint of fixed angle. The stretching modulus of an individual fiber is given by $K_{\mathrm{s}}=E A$, where $E$ is the Young's modulus and $A$ is the cross-sectional area. The Young's modulus of a fiber in aqueous conditions has been estimated to be between 30 and $800 \mathrm{MPa}$ [23-25], and we use a modulus of $50 \mathrm{MPa}$, which fits the data well and is also close to the value chosen by Stylianopoulous and Barocas (i.e., $79 \mathrm{MPa}$ ) to fit their model [21]. It has been shown that a single fiber will stiffen by a factor of 2-4 when strained [25]. We choose here to use a constant $E$ both to reduce the number of parameters in the model and to see if geometric reorientation of the network is enough to explain strain stiffening. Stylianopoulos and Barocas [21] also explored the bilinear and exponential constitutive relations for the individual fibers and observed only minor effects on the macroscopic network behavior. The radius of each fiber is $r=30 \mathrm{~nm}[3,22]$. The bending modulus of the fiber is given by $K_{\mathrm{b}}=E I=32 \mathrm{pN}-\mu \mathrm{m}^{2}$, where $I=\pi r^{4} / 4$ [26]. No crosslinking agent has been added to the gel, and very little is known about the nature of the naturally formed collagen crosslinks. We find that we can fit all the data by setting the torsional spring stiffness to $K_{\mathrm{x}}=300 \mathrm{pN}-\mu \mathrm{m}$. To compare $K_{\mathrm{b}}$ to $K_{\mathrm{x}}$, we consider $K_{\mathrm{b}} / l_{\mathrm{c}}$, where the mean crosslink spacing is given by $l_{\mathrm{c}} \sim 2 \mu \mathrm{m}$ [3]. Thus, we find that $K_{\mathrm{x}} \sim 20 K_{\mathrm{b}} / l_{\mathrm{c}}$. One possible reason for an increase in stiffness at the crosslinks could be an increase in fiber radius near the crosslinks by a factor of 2, as bending stiffness scales by $r^{4}$. However, much is still unknown about the internal structural of the fibrils and the interfiril and intrafibril bonding mechanics, and therefore, there is necessarily no reason to expect $K_{\mathrm{x}}$ and $K_{\mathrm{b}} / l_{\mathrm{c}}$ to be equivalent.

We assume that in the undeformed state of the network, there are no internal stresses. Thus, the fibers have an innate curvature and the crosslinks have an equilibrium angle equal to that in their initial configuration. We ignore entropic contributions to the fiber mechanics. While the geometric persistence length of these fibers has been measured to be $20 \mu \mathrm{m} \mathrm{[3],} \mathrm{the} \mathrm{thermal} \mathrm{persistence} \mathrm{length} \mathrm{is} \mathrm{much} \mathrm{longer}$ $l_{\mathrm{p}}=K_{\mathrm{b}} / k T \sim 1 \mathrm{~cm}$. Furthermore, in the case that the strain stiffening is dominated by thermal compliance, one would expect to see a decrease in the yield strain with increasing concentration [27]. Collagen gels, however, have been shown to have a constant yield strain of about $60 \%$ for a wide range of concentrations [28]. The total energy in the network for a given configuration is given below.

$$
U=\sum_{i=1}^{N_{\mathrm{s}}} \frac{K_{\mathrm{s}}}{L^{i}} \frac{\left(\Delta L^{i}\right)^{2}}{2}+\sum_{i=1}^{N_{\mathrm{b}}} \frac{K_{\mathrm{b}}}{L^{i}} \frac{\left(\Delta \theta_{\mathrm{b}}^{i}\right)^{2}}{2}+\sum_{k=1}^{N_{\mathrm{x}}} K_{\mathrm{x}} \frac{\left(\Delta \theta_{\mathrm{x}}^{i}\right)^{2}}{2}
$$

Here $N_{a}$ is the number of elements of type $a \in\{\mathrm{s}, \mathrm{b}, \mathrm{x}\}$, which denotes stretching, bending, and crosslink, $L_{i}$ is the length of stretching element, $\theta_{\mathrm{b}}^{i}$ and $\theta_{\mathrm{x}}^{i}$ are the bending and crosslink angles, respectively, and $\Delta$ indicates the difference between the deformed and undeformed state.

To calculate the stress-strain relationship of our model network, we performed a series of 18 incremental strain steps by imposing a small deformation on one face, $F_{1}$, while holding the opposite face, $F_{0}$, fixed. We impose two types of deformations: tension [Figure 1(d)] and shear [Figure 1(e)]. In a tensile deformation, we allow the vertices on $F_{0}$ and $F_{1}$ to move freely in directions perpendicular to the imposed strain to allow for perpendicular contraction, which is seen to occur in these experiments $[28,29]$. In experiments of this type, the distance between $F_{0}$ and $F_{1}$ is on the order of centimeters, and the simulated network represents a small region near the center of a sample. In shear, we do not allow the boundary nodes on $F_{0}$ and $F_{1}$ to move freely. We compare the shear results to cone-plate rheometer experiments, where the shear faces are bound to the rheometer. Here, the distance between $F_{0}$ and $F_{1}$ is $109 \mu \mathrm{m}$, and the simulated network is one fourth the length of the experimental sample between the boundaries. In both deformations, all other nodes, including those on the four remaining faces of the network, are free to move. The minimum energy state of the network at an imposed strain $U\left(\epsilon_{i j}\right)$ is found using a conjugate gradient method developed by Hager and Zhang [30]. The stress required to hold the network in its current configuration is given by $\sigma_{i j}\left(\epsilon_{i j}\right)=\left(d U / d \epsilon_{i j}\right) / A$, where $A$ denotes the area of $F_{0}$.

\section{RESULTS}

The results are averaged over four extracted networks and over all six shear deformations $\left.\epsilon_{i j}\right|_{i \neq j}$ in the sheared network and all three principal tensile directions $\epsilon_{i i}$ in the stretched network. The stress-strain relationship for a single gel is shown in Figure 1(f), and the results for all gels are summarized in Figure 2. Below $\sim 5 \%$ strain amplitude, the material deforms primarily in an elastic fashion, but above $\sim 5 \%$, the material deforms irreversibly. Although the gel can recover some of its initial material properties given sufficient time to relax, it never completely recovers. In Figure 3(a), we plot the small strain modulus from the samples by calculating the slope of the stress-strain curve at small strains (0.2-2\%). In Figure 3(b), we compare the previously reported tensile modulus of large samples that are centimeters in length [28] to the simulation. Here, in both simulation and experiment, the large strain modulus is computed by fitting lines to the stressstrain curve in the regime of $20-40 \%$ strain. At small strains, 


\section{FIGURE 2}

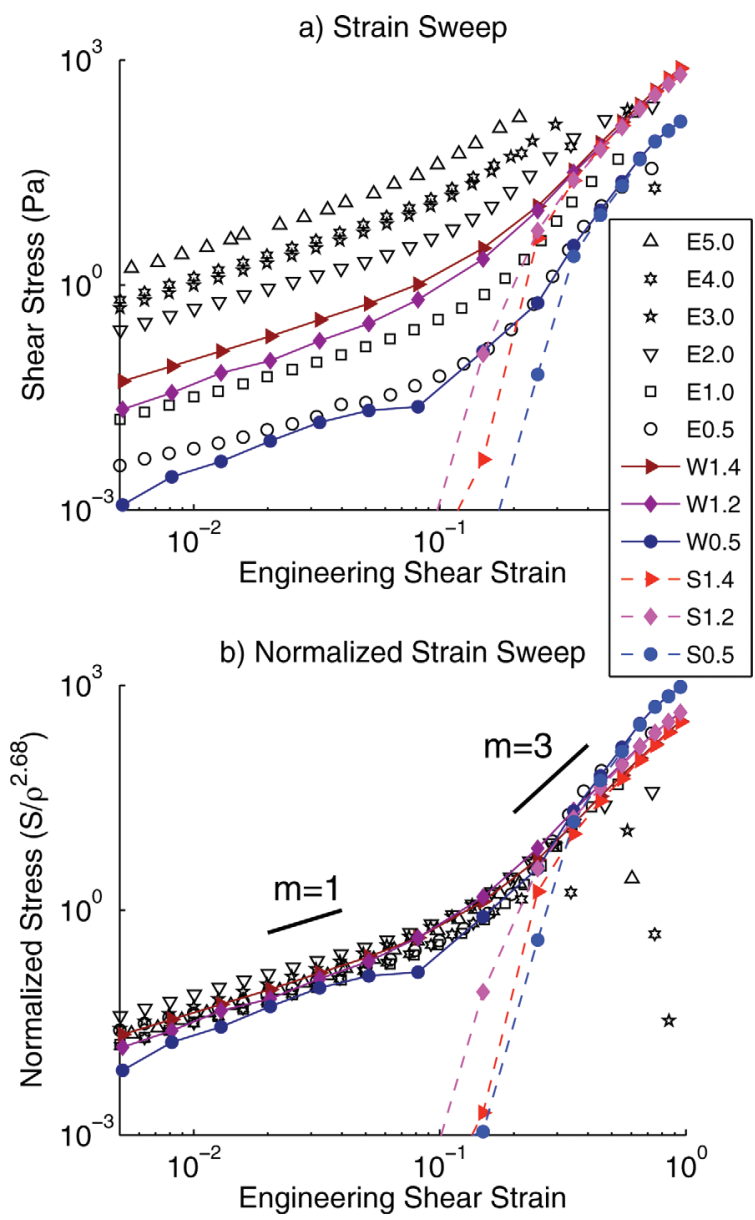

Stress-strain sweeps of the gel, from 0.5 to $100 \%$ strain. In the artwork, $\mathrm{E}$ denotes experiment, $\mathrm{W}$ denotes the elastic beam model, $\mathrm{S}$ denotes the spring model where we set $K_{\mathrm{x}}=K_{\mathrm{b}}=0$, and the number denotes the collagen density in $\mathrm{mg} / \mathrm{mL}$. (a) Unscaled results. Note the good agreement between model and data at small and large strains. (b) When the curves are scaled by $\rho^{2.68}$, relatively good data collapse is achieved. We denote lines of slope $m=1$ and $m=3$ to guide the eye. At large strains, scaling breaks down and the low-density curves overtake the high-density curves, because at large strains, stiffness scales linearly with density. Thus, this rescaling mainly serves as a visualization tool and does not represent a true data collapse.

the stress-strain response is linear, as expected, and at larger shear strains, the stress-strain response stiffens considerably. In Figure 3(a), we show that the small strain modulus scales by $\sigma_{12} \sim \rho^{2.68} \epsilon_{12}$, where $\rho$ is the collagen density. At this time, it is not possible to verify the power law scaling in the model as only densities of $0.5,1.2$, and 1.4 were observed. The fluorescent labeling of the network changes the polymerization properties of the network, causing it to clump at higher densities. We use this scaling relationship to collapse the curves in
Figure 2(b). The close agreement between model and experiment indicates that strain stiffening due to the geometric rearrangement of the collagen fibers is enough to explain the strain stiffening seen in experiments.

At large strains ( $\sim 50 \%$ in tension and $\sim 200 \%$ in shear), the stress-strain curve of the model becomes linear again, although with a much steeper slope. In Figure 3(b), we compare the large strain tensile behavior of the model to the experiments of Roeder et al. [28]. Although our model underestimates their experimental measurement by a factor of 2.5 , we find this to be reasonable as the two experiments used different collagen protocols. In particular, different buffers were used. In Figure 2, we also explore the case where $K_{\mathrm{x}}=K_{\mathrm{b}}=0$, such that we have only a network of springs connected at freely rotating pin joints. At low strains, the network can be deformed without exerting any stress, but at strains higher

\section{FIEURE 3}

(a)

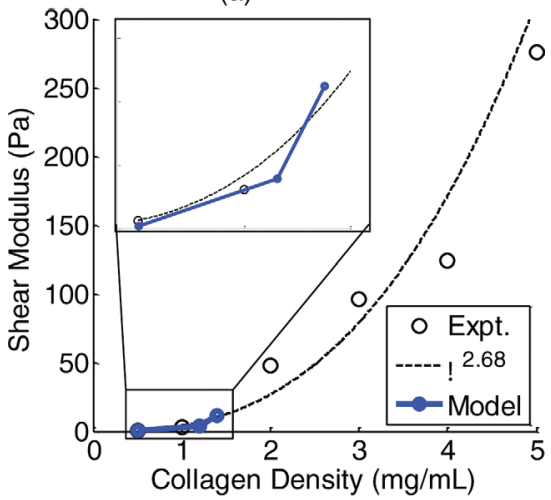

(b)

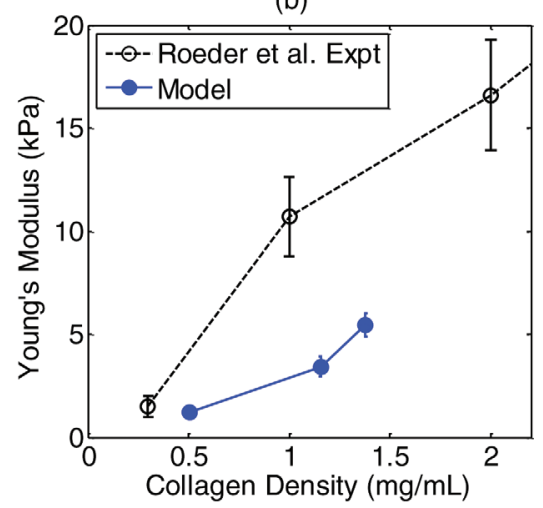

(a) The small strain shear modulus is compared with the cone-plate rheometer experiments, and a scaling law of $G^{\prime} \sim \rho^{2.68}$ is observed for the experiment. (b) The large strain tensile modulus is from the model and from the experiments of Roeder et al. [28]. Results differ by a factor of 2.5 , which is reasonable as the two experimental protocols were different [28]. 


\section{FIGURE 4}

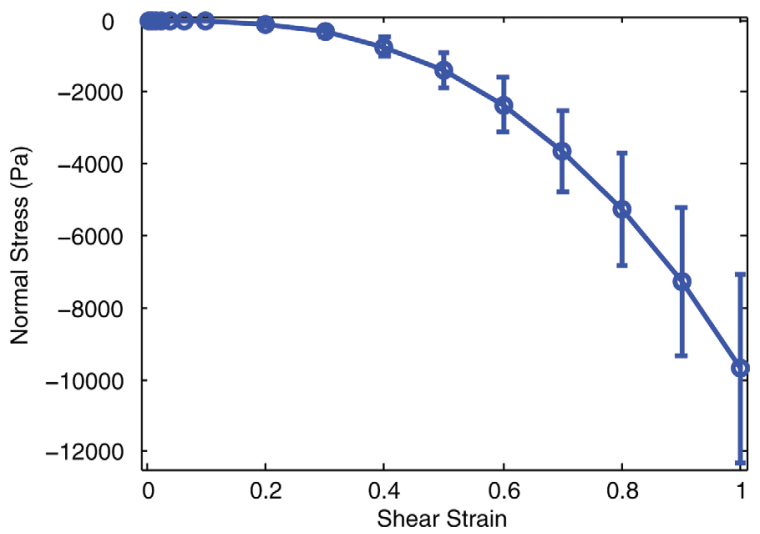

Average negative normal stresses for the $1.2 \mathrm{mg} / \mathrm{ml}$ gel in response to shear.

than $25 \%$, we see that this simplification adequately describes the gel.

A topic of investigation explored by many is the validity of the assumption that these networks deform affinely $[14,15]$. For brevity, we only state that the deformations are highly nonaffine at small strains where the majority of the energy is stored in bending rather than stretching. This is best seen in Figure 2. Affine deformations have the property that the majority of the energy stored in the network is in stretching the fibers, but here, fiber stretching makes no contribution to the network stiffness until strains are greater than $10 \%$.

In response to shear stress, the simulated networks also exhibited negative normal stresses that grow quadratically with strain (Figure 4), as observed in the experiments of Janmey et al. [9]. We also examined the perpendicular contraction of the gel in response to axial strain in comparison with the experiments of Vader et al. [11] and Roeder et al. [4], as shown in Figure 5. The perpendicular strain was taken to be the perpendicular displacement divided by the original perpendicular distance from the origin averaged over all the nodes. Here, the model does not match the experiments well. In particular, in both experiments, the $z$-compression is significantly greater than the $y$-compression. By contrast, in the simulation, the $z$ - and $y$-compression are almost overlapping. Furthermore, both experiments exhibited considerably more perpendicular compression than seen in the simulation. One possible explanation is that the geometry of the experiments done here (sheared cone-plate rheometer) is considerably different from that in the other experiments (stretched disk and stretched 3D I-beam). Another possibility is that the FIRE network extraction algorithm overestimates the number of crosslinks of the network. It may also be that some of the crosslinks in the network slip, thus allowing the network to further compress in response to axial tension. To give the same strain-stiffening behavior, this would require stiffer crosslinks. Further exploration, however, was beyond the scope of this article. Despite the lack of quantitative agreement, we note that nonlinearly increasing perpendicular strain is qualitatively similar to that observed in Vader et al. [11].

\section{SUMMARY AND DISCUSSION}

In summary, we have presented a microstructural model of a $3 \mathrm{D}$ biopolymer gel using a network geometry that is based on the true network architecture. It differs from previous work in that we use realistic network architectures that have been extracted using the FIRE algorithm. We specifically focus on the mechanics of collagen-I networks, but we emphasize that this modeling approach is generalizable to other biopolymer networks. The model has three parameters: $\left\{E, r, K_{\mathrm{x}}\right\}$. The fiber radius and tensile modulus can be measured experimentally, and the model uses realistic parameters. The crosslink torsional spring constant must be fit to the data and we used $K_{\mathrm{x}} \sim 20 K_{\mathrm{b}} / l_{\mathrm{c}}$. Fitting this single parameter gives the right strain-stiffening behavior for networks at three different densities at strains that vary from 0.5 to $50 \%$. This result lends support to the hypothesis put forward by Onck et al. [15] that strain stiffening in polymer networks and particularly collagen-I gels is governed by rearrangement of the gel. Here, we note that the strain-stiffening mechanisms for collagen are likely different than for other gels. Because of its long persistence length $(1 \mathrm{~cm})$ fibers, the effects of entropic stiffening in these gels is known to be minimal. Therefore, the entropic stiffening hypothesis [31] is not applicable here. However, for other biopolymer gels such as actin, where the

\section{FIGURE 5}

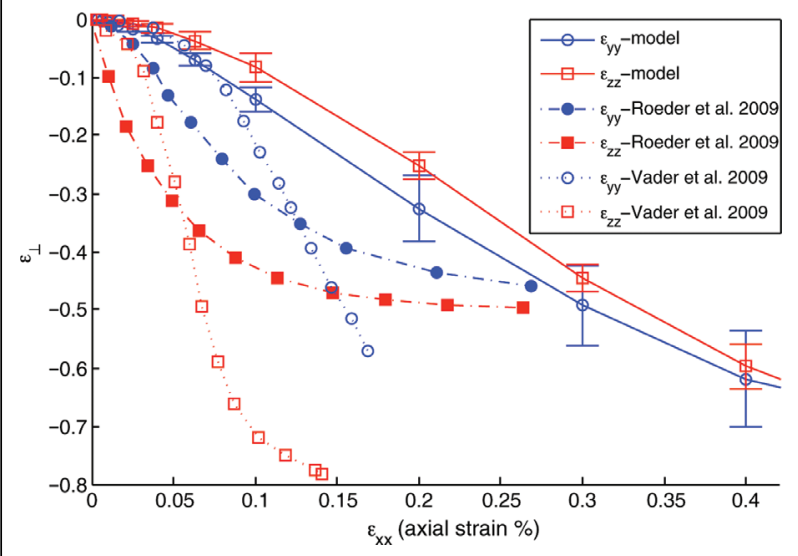

Perpendicular strains for the $1.2 \mathrm{mg} / \mathrm{mL}$ gel in response to tension when compared with the experiments from Vader et al. [11] and Roeder et al. [4]. 
persistence length of the filaments is in the order of the mesh size, entropic stiffening of individual fibers is more likely to be an important component that contributes to strain stiffening. The stiffening in fibrin clots may depend not only on geometric rearrangements but also on the unfolding of the fibrin protein [32].

Another finding of the model is that at shear strains greater than $25 \%$, the stiffness of the gel is governed almost entirely by stretching of the fibers. This result is relevant for collagen because cells embedded in these gels are seen to produce deformations of this order of magnitude [33]. In modeling large systems of this type where the strains are large, it may be sufficient to treat each fiber as a spring rather than an elastic beam to reduce the computation time. This work also demonstrates that an understand of the crosslink mechanics in these systems is critical to understand their mechanical properties, as has been seen previously [34]. In much of the theoretical work that has been done on random stick networks, the crosslinks are treated either as freely rotating pin joints or welded joints of fixed angle [14, 15, 27]. Although these are sensible simplifying assumptions in developing a theory, they are not adequate for describing actual networks.

The mechanical properties of an individual fiber depends on the fiber microstructure and ranges from relatively linear elastic behavior even at large strains [24] to moderate stiffening [35]. Here, we note that the simulated large strain modulus underestimated the actual large strain modulus by a factor of two. This difference could potentially be explained by a stiffening of the individual fibers by a factor of two at large strains, but confirmation would require additional experiments measuring the stress-strain properties of individual fibers in these collagen gels.

We note that this model has been designed to capture the intermediate time scale behavior of the network (minutes to hours), where the network behaves as an elastic solid. The viscous modulus is known to be a factor of 10 less than the elastic modulus within the range of $0.1-100 \mathrm{~Hz}[36,37]$, but at even faster time scales, the viscosity of the fluid will play a critical role. At slower time scales, the assumption that the crosslinks remain relatively fixed [36] breaks down as plastic deformation and creep occur. This simplified model provides a starting point in the development of a more complete model of collagen gel. Ultimately, a more sophisticated approach, such as that taken by Rodney et al. [38] will be necessary to capture the full dynamic behavior of the gel, where crosslinks are allowed to slip and break.

\section{ACKNOWLEDGEMENTS}

The authors thank V. Barocas, T. Stylianopoulos, E. A. Sander, E. Tuzel, H. Zhang, H. Othmer, T. Jackson, P. Smereka, R. Krasny, F. MacKintosh, A. Kabla, R. Lee, M. Dewberry, L. Kaufman, and L. Jawerth, for their discussions. This work was supported by NIH Bioengineering Research Partnership (grant R01 CA085139-01A2) and the Institute for Mathematics and its Applications.

\section{REFERENCES}

1. Lodish, H.; Berk, A.; Zipursky, S.L.; Matsudaira, P.; Baltimore, D.; Darnell, J. Molecular Cell Biology; W. H. Freeman and Company: New York, 1999.

2. Fung, Y.C. Biomechanics: Mechanical Properties of Living Tissues, 2nd ed.; Springer: New York, 1993.

3. Stein, A.M.; Vader, D.A.; Jawerth, L.M.; Weitz, D.A.; Sander, L.M. An algorithm for extracting the network geometry of 3D collagen gels. J Microsc 2008, 232, 463-475.

4. Roeder, B.; Kokini, K.; Voytik-Harbin, S. Fibril microstructure affects strain transmission within collagen extracellular matrices. J Biomech Eng 2009, 131, 031004.

5. Chandran, P.L.; Barocas, V.H. Microstructural mechanics of collagen gels in confined compression: Poroelasticity, viscoelasticity, and collapse. J Biomech Eng 2004, 126, 152-166.

6. Friedl, P.; Zanker, K.S.; Brocker, E.-B. Cell migration strategies in 3-D extracellular matrix: Differences in morphology, cell matrix interactions, and integrin function. Microsc Res Tech 1998, 43, 369-378.

7. Kaufman, L.J.; Brangwynne, C.P.; Kasza, K.E.; Filippidi, E.; Gordon, V.D.; Deisboeck, T.S.; Weitz, D.A. Glioma expansion in collagen i matrices: Analyzing collagen concentration-dependent growth and motility patterns. Biophys $\mathrm{J} 2005,89,635-650$.

8. Stein, A.M.; Demuth, T.; Mobley, D.; Berens, M.E.; Sander, L.M. A mathematical model of glioblastoma tumor spheroid invasion in a three-dimensional in vitro experiment. Biophys J 2007, 92, 356-365.

9. Janmey, P.; McCormick, M.E.; Rammensee, S.; Leight, J.L.; Georges, P.C.; MacKintosh, F. Negative normal stress in semiflexible biopolymer gels. Nat Mater 2006, 6, 48-51.

10. Kabla, A.; Mahadevan, L. Nonlinear mechanics of soft fibrous networks. J R Soc Interface 2007, 4, 99-106.

11. Vader, D.; Kabla, A.; Weitz, D.; Mahadevan, L. Strain-induced alignment in collagen gels. PloS One e5902, 2009, 4, 1-12.

12. Storm, C.; Patsore, J.; MacKintosh, F.; Lubensky, T.; Janmey, P. Nonlinear elasticity in biological gels. Nature 2005, 435, 191-194.

13. Heussinger, C.; Frey, E. Stiff polymers, foams, and fiber networks. Phys Rev Lett 2006, 96, 017802.

14. Heussinger, C.; Schaefer, B.; Frey, E. Nonaffine rubber elasticity for stiff polymer networks. Phys Rev E 2007, 76, 031906.

15. Onck, P.; Koeman, T.; van Dillen, T.; van der Giessen, E. Alternative explanation of stiffening in cross-linked semiflexible networks. Phys Rev Lett 2005, 95, 178102.

16. Huisman, E.M.; van Dillen, T.; Onck, P.; van der Giessen, E. Three-dimensional cross-linked f-actin networks: Relation between network architecture and mechanical behavior. Phys Rev Lett 2007, 99, 208103. 
17. Head, D.; MacKintosh, F.; Levine, A.J. Nonuniversality of elastic exponents in random bond-bending networks. Phys Rev E 2003, 68, 025101(R).

18. Wilhelm, J.; Frey, E. Elasticity of stiff polymer networks. Phys Rev E 2003, 91, 108103.

19. Yang, Y.; Kaufman, L. Rheology and confocal reflectance microscopy as probes of mechanical properties and structure during collagen and collagen/hyaluronan self-assembly. Biophys J 2009, 96, 1566-1585.

20. Chandran, P.L.; Barocas, V.H. Affine versus non-affine fibril kinematics in collagen networks: Theoretical studies of network behavior. J Biomech Eng 2006, 128, 259-269.

21. Stylianopoulos, T.; Barocas, V.H. Volume-averaging theory for the study of the mechanics of collagen networks. Comput Methods Appl Mech Eng 2007, 196, 2981-2990.

22. Raub, C.B.; Suresh, V.; Krasieva, T.; Lyubovitsky, J.; Mih, J.D.; Putnam, A.J.; Tromberg, B.J.; George, S.C. Noninvasive assessment of collagen gel microstructure and mechanics using multiphoton microscopy. Biophys J 2007, 92, 2212-2222.

23. Graham, J.S.; Vomund, A.N.; Phillips, C.L.; Grandbois, M. Structural changes in human type I collagen fibrils investigated by force spectroscopy. Exp Cell Res 2006, 299, 335-342.

24. Miyazaki, H.; Hayashi, K. Tensile tests of collagen fibers obtained from the rabbit patellar tendon. Biomed Microdevices 1999, 2, 151-157.

25. van der Rijt, J.A.J.; van der Werf, K.0.; Bennink, M.L.; Dijkstra, P.J.; Feijen, J. Micromechanical testing of individual collagen fibrils. Macromol Biosci 2006 , $6,697-702$.

26. Yang, L.; van der Werf, K.0.; Koopman, B.F.; Subramaniam, V.; Bennink, M.L.; Dijkstra, P.J.; Feijen, J. Mechanical properties of native and cross-linked type i collagen fibrils. Biophys J 2008, 94, 2204-2211.

27. Head, D.; Levine, A.J.; MacKintosh, F. Distinct regimes of elastic response and deformation modes of cross-linked cytoskeletal and semiflexible polymer networks. Phys Rev E 2003, 68, 061907.

28. Roeder, B.A.; Kokini, K.; Sturgis, J.E.; Robinson, J.P.; Voytik-Harbin, S.L. Tensile mechanical properties of three-dimensional type i collagen extracellular matrices with varied microstructure. J Biomech Eng 2002, 124, 214-222.

29. Krishnan, L.; Weiss, J.A.; Wessman, M.D.; Hoying, J.B. Design and application of a test system for viscoelastic characterization of collagen gels. Tissue Eng 2004, 10, 241-252.

30. Hager, W.W.; Zhang, H. A new conjugate gradient method with guaranteed descent and an efficient line search. SIAM J 0ptim 2005, 16, 170-192.

31. MacKintosh, F.; Kas, J.; Janmey, P. Elasticity of semiflexible biopolymer networks. Phys Rev Lett 1995, 75, 4425-4428.

32. Brown, A.; Litvinov, R.; Discher, D.; Purohit, P.; Weisel, J. Multiscale mechanics of fibrin polymer: Gel stretching with protein unfolding and loss of water. Science 2009, 325, 741-744.

33. Pizzo,A.M.; Kokini, K.;Vaughn, L.C.; Waisner, B.Z.; Voytik-Harbin, S.L. Extracellular matrix microstructural composition regulates local cell-ecm biomechanics and fundamental fibroblast behavior: A multidimensional perspective. J Appl Physiol 2005, 98, 1909-1921.

34. Wagner, B.; Tharmann, R.; Haase, I.; Fischer, M.; Bausch, A.R. Cytoskeletal polymer networks: The molecular structure of cross-linkers determines macroscopic properties. Proc Natl Acad Sci USA 2006, 103, 13974-13978.

35. Gentleman, E.; Lay, A.N.; Dickerson, D.A.; Nauman, E.A.; Livesay, G.A.; Dee, K.C. Mechanical characterization of collagen fibers and scaffolds for tissue engineering. Biomaterials 2003, 24, 3805-3813.

36. Barocas, V.H.; Moon, A.G.; Tranquillo, R.T. The fibroblast-populated microsphere assay of cell traction force-part2. measurement of the cell traction coefficient. J Biomech Eng 1995, 117, 161-170.

37. Velegol, D.; Lanni, F. Cell traction forces on soft biomaterials. I. microrheology of type i collagen gels. Biophys J 2001, 81, 1786-1792.

38. Rodney, D.; Fivel, M.; Dendievel, R. Discrete modeling of the mechanics of entangled materials. Phys Rev Lett 2005, 95, 108004. 